\title{
Photoelectron spectroscopy of jet-cooled aluminium cluster anions
}

\author{
G. Ganteför, M. Gausa, K.H. Meiwes-Broer, and H.O. Lutz \\ Fakultät für Physik, Universität Bielefeld, D-4800 Bielefeld 1, Federal Republic of Germany
}

\begin{abstract}
Aluminium cluster anions $\left(\mathrm{Al}_{n}^{-}\right)$are produced by laser vaporization without additional ionization and cooled by supersonic expansion. Photoelectrons from mass-identified anion bunches $(n=2 \ldots 25)$ are detached by laser light $(h \nu=3.68 \mathrm{eV})$ and undergo energy analysis in a magnetic bottle-type time-of-flight spectrometer. The measurements provide information about the electronic excitation energies from ionic ground states to neutral states of the clusters. In contrast to bulk aluminium these cluster photoelectron spectra partially have well-resolved bands which originate from low-lying excited bands. For small clusters, especially the aluminium dimer and trimer, quantum-chemical calculations will be compared to the measurements. The electron affinity size dependence of larger clusters shows conclusive evidence for "shell" effects.
\end{abstract}

PACS: $36.40 .+\mathrm{d} ; 33.60 . \mathrm{Cv}$

\section{Introduction}

Atomic clusters provide a link between the isolated atom/molecule and the "infinite" condensed state. Aside from considerable applied potential (catalysis, nucleation, thin film deposition) they are thus of great basic interest. At present evidence exists for a number of properties of small metal clusters being quite different from those of respective bulk systems. The convergence to bulk behaviour depends, of course, on the investigated material and on the physical character which is interrogated. In restricting the discussion to aluminium clusters, we find thorough investigations of their physics and chemistry: Already mass spectra obtained with different methods like sputtering, thermal evaporation or laser vaporization, allow a first glance into their electronic behaviour and the binding situation [1-4]. Other areas of interest are collisions and chemical reactions of $\mathrm{Al}_{n}[2,5]$, magnetic properties [3], the unimolecular decay behaviour $[1,6]$ and, most recently, their photoelectron spectroscopy $[7,8]$.

Aluminium as the second member in Group III in the periodic table is the first metallic element which contains a $p$-valence electron. The interaction be- tween $s$ - and $p$-like orbitals in the cluster can be expected to give rise to a set of electronic levels which are different from those of alkali-like systems. In several calculations concerning the geometric structure and electronic behaviour the complexity of aluminium clusters is apparent [9-13], thus raising the question about the role of low-lying excited states or isomers. Ultraviolet photoelectron spectra, as presented in this paper, might show a way to get insight into the electronic behaviour of these species.

\section{Experimental}

The experimental set-up is shown schematically in Fig. 1. It consists of a laser evaporation source, a pulsed acceleration system with time-of-flight (TOF) arrangement, a photo-ionisation chamber served by a pulsed UV laser, and a magnetic bottle-type TOF electron spectrometer. The differentially pumped vacuum system allows cluster source operation at an average pressure of about $10^{-4} \mathrm{mbar}$, collisional-free acceleration of the cluster ions at $10^{-6} \mathrm{mbar}$, and photodetachment under ultra-high vacuum $\left(<10^{-8}\right.$ mbar $)$. 


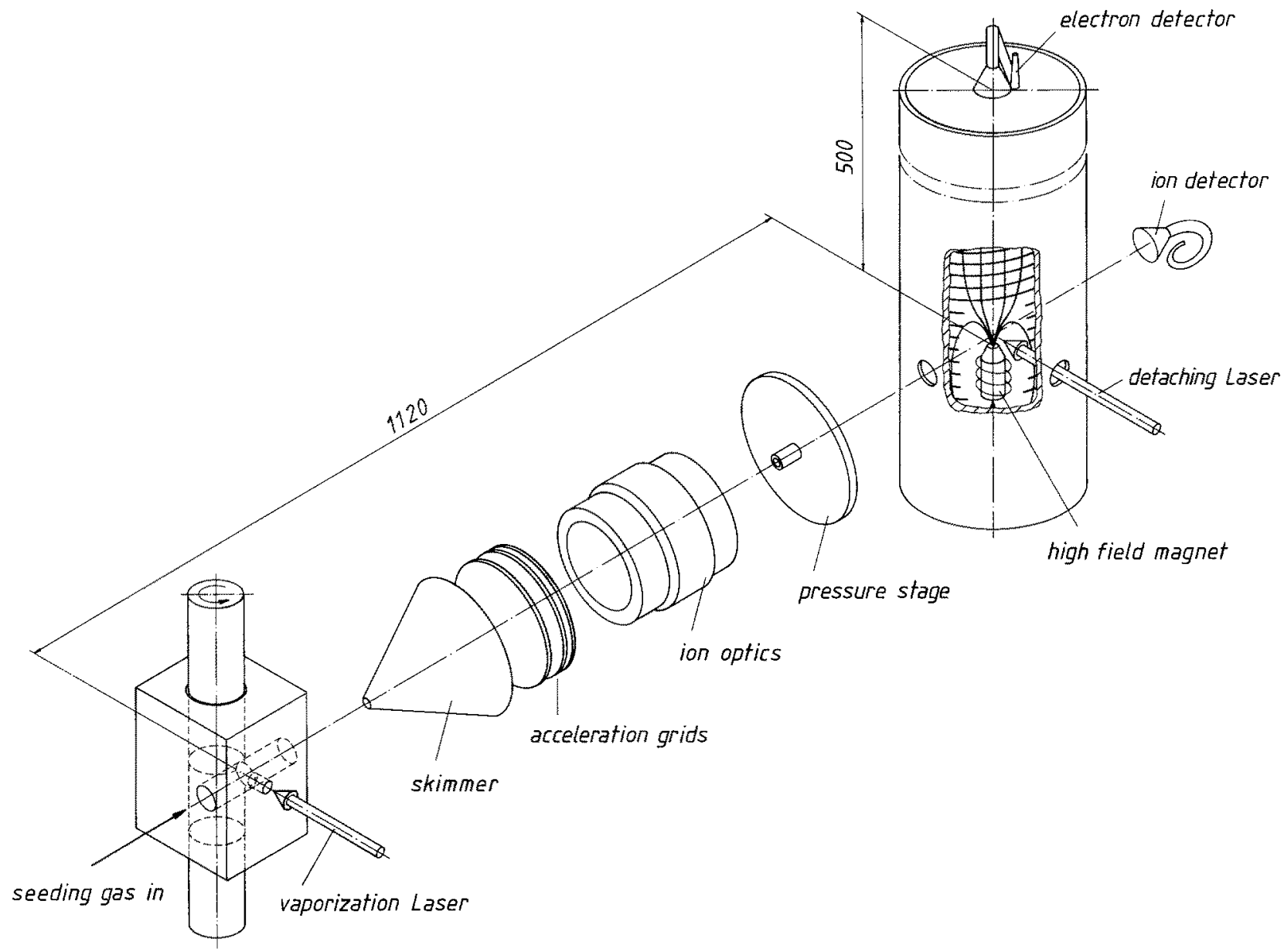

Fig. 1. Schematic diagram of the experimental setup: Cold metal cluster anions are produced by laser vaporization and cooled by supersonic expansion (left). After pulse formation electrons are detached from mass-identified cluster bunches and undergo energy analysis in a magnetic bottle spectrometer (right)

\section{A. Cluster ion source and TOF arrangement}

The cluster source employs laser vaporization of a target rod of the desired metal mounted in a pulsed, high pressure supersonic nozzle $[4,14]$. Different types of pulsed gas valves (piezo or magnetically driven) produce $\mathrm{He}$ or $\mathrm{Ne}$ pulses at backing pressures of $2 \ldots 15$ bar. Target evaporation is effected by (pulsed) excimer laser light ( $\mathrm{ArF}, \mathrm{XeCl}, 100 \mathrm{~mJ} /$ pulse), producing an intense plasma which is flushed by the carrier gas pulse through a channel of variable length into high vacuum. A number of channel designs (diameter $0.5 \ldots 2 \mathrm{~mm}$, length $5 \ldots 50 \mathrm{~mm}$ ) and nozzle geometries (cylindrical, conical) has been tested to optimize the intensity of (positively and negatively charged) clusters which emerge directly from the source without additional ionization. A detailed discussion of the influence of source conditions upon mass spectra and ion energy distributions will be published

Table 1. Source parameters for the production of aluminium cluster anions

Target material

Vaporizing light

Carrier gas

Gas valve

Backing pressure

Channel design

Nozzle

Target rotation

Distance nozzle skimmer

Source chamber volume

Repetition rate
Aluminum rod $99.95 \%, 5 \mathrm{~mm} \varnothing$

Excimer laser $308 \mathrm{~nm}$,

$100 \mathrm{~mJ} /$ pulse,

spot size $0.5 * 1 \mathrm{~mm}$

Helium $99.99 \%$

General valve, series 9 (magnetically driven); pulse duration $0.5 \mathrm{~ms}$

8 bar

$\varnothing 1.5 \mathrm{~mm}$, length $5 \mathrm{~mm}$ conical $\left(20^{\circ}\right)$, length $30 \mathrm{~mm}$

$0 . .0 .5 \mathrm{rpm}$

$110 \mathrm{~mm}$

$80 \mathrm{ltr}$, oil-pumped $(1500 \mathrm{l} / \mathrm{s})$ up to $120 \mathrm{~Hz}$, typically $80 \mathrm{~Hz}$ 
elsewhere [15]. Table 1 gives some experimental parameters used for aluminium anion production.

The laser-produced plasma jet emerging from the nozzle traverses the source chamber which is carefully kept free of electric fields in order not to disturb the supersonic expansion. As a consequence the plasma beam contains positive and negative cluster ions when it enters the accelerator stage behind the skimmer. By its polarity, this two-stage pulsed acceleration system [7] determines the selected clusters's charge state and forms a short cluster packet of defined energy. Its composition is analyzed in a TOF mass spectrometer operating colinearly with the expansion direction (see Fig. 1). At the end of the drift region, the ions are detected by a channeltron and registered in a transient digitizer (Transiac 4001). The experiment can be operated at a repetition rate of up to $120 \mathrm{~Hz}$.

\section{B. Magnetic bottle electron spectrometer}

The cluster packets are spatially and temporally focussed into the starting area of a TOF electron spectrometer where they are hit by the light pulse of a nitrogen laser $(h v=3.68 \mathrm{eV}$, pulse length $10 \mathrm{~ns})$. In principle, this device can work without any magnetic fields as has been demonstrated by Posey et al. [16]. Of course, under such conditions only electrons emitted into a small solid angle (approx. $10^{-4}$ sterad) will be detected; in addition, careful magnetic shielding is necessary. Alternatively, Kruit et al. [17] suggested a magnetic bottle-type spectrometer in which the electrons are born in a strong magnetic field, and subsequently pass adiabatically into a weak-field zone which guides them into the detector. A solid acceptance angle of $2 \pi$ is theoretically possible. Our spectrometer is based on this idea; the high field (approx. 400 gauss in the detachment zone) is produced by an electromagnet with iron core, the guiding field ( 5 gauss) is produced by a long coil; the whole electron spectrometer is surrounded by a $\mu$-metal shield. The electrons are detected after a drift distance of $0.5 \mathrm{~m}$ by a channeltron, the flight times being recorded with a $100 \mathrm{MHz}$ transient digitizer and added in a fast memory. With an intense cluster bunch up to 10 photoelectrons per laser shot may be analyzed. Under normal operation, however, the power of the detaching laser is reduced (approx. $10^{6} \mathrm{~W} / \mathrm{cm}^{2}$ ) in order to avoid multiphoton processes in the cluster ions as well as in the residual gas. Therefore, typical $\mathrm{PE}$ currents are $0.1-1$ electrons/shot at a repetition rate of about $80 \mathrm{~Hz}$.

The photoelectron kinetic energies are defined by

$E_{\mathrm{kin}}=h v-I_{1,2}-\Delta E_{\mathrm{vib}}-\Delta E_{\mathrm{rot}} \pm \Delta E_{\mathrm{trans}}$
$I_{1,2}$ : difference between the electronic states before and after electron detachment

$\Delta E_{\mathrm{vib}}, \Delta E_{\mathrm{rot}}, \Delta E_{\mathrm{trans}}:$ change of vibrational, rotational, and translational energy upon electron emission.

Uncertainties in surface potential, space charge, and the geometry and operating conditions of the magnetic bottle can contribute to possible errors and/ or limit the spectrometer resolution. Surface potentials may be up to some hundreds of $\mathrm{meV}$, and are mainly due to surface contamination in the detachment zone. Their influence can be reduced by ensuring ultra-high vacuum conditions. This is prerequisite in particular for PES with photon energies above about $5 \mathrm{eV}$ (to be published). Space charge effects may arise either in case of high photoelectron currents, or from the remaining negative ion cloud interacting with the departing electrons. Such effects can be probed by looking for shifts of the electron energy as a function of laser power and cluster ion intensity; for our experimental conditions none of these could be observed.

The magnetic bottle spectrometer has been thoroughly discussed by Kruit et al. [17]. For a photoionised cluster at rest the degree of electron trajectory parallelisation is given by the ratio of the high $\left(B_{h}\right)$ and low $\left(B_{l}\right)$ magnetic field. Angular momentum conservation determines the maximum angle $\theta$ between the spectrometer axis and the helical motion in the weak field; it is given by [17]

$\theta=\arcsin B_{l} / B_{h}$.

The intrinsic energy resolution - based on the final angle $\theta$ - can be approximated by $\triangle E / E \approx B_{L} / B_{h}$. The major contribution to $\Delta E / E$ stems from the high field region where the electron trajectories have not yet been parallelized; an increase of the drift tube length leads to a decrease of this effect. The energy resolution is further limited by off-axis ionization of the spatially extended cluster packet; in addition, the electron angular distribution (influenced, in particular, by the laser polarization) may change the peak shape drastically. Furthermore, we note a strong dependence of the energy resolution on the cluster velocity; the same observation has recently been reported by other authors [19]. In contrast to Kruit et al. [17] who ionized (neutral) atoms at thermal velocities, our clusters are fairly fast (about $10^{4} \mathrm{~m} / \mathrm{s}$ ) and thus give rise to noticeable Doppler broadening. To reduce this effect the cluster ion energy has to be kept as low as possible. Our colinear cluster source arrangement allows operation at low beam energies (down to $20 \mathrm{eV}$ ), and no special deceleration device is needed to work under nearly Doppler-free conditions.

Different magnet geometries and electron drift tube lengths have been tested. The $\mathrm{Al}_{n}^{-} \mathrm{PE}$ spectra 
presented here were recorded with a short $(0.5 \mathrm{~m})$ drift tube and a simple electromagnet with an iron core $\left(B_{h} / B_{l}=80: 1\right)$ at an overall energy resolution of $\Delta E /$ $E \approx 0.1$. A longer drift tube $(2 \mathrm{~m})$ and a stronger highfield magnet has also been used and turned out to yield an improved energy resolution; resulting $\mathrm{PE}$ spectra of silver, nickel and tin cluster anions are described elsewhere [18].

\section{Results}

Figure $2 \mathrm{a}, \mathrm{b}$ shows $\mathrm{Al}_{n}^{-}$mass spectra at a cluster beam energy of $300 \mathrm{eV}$. In the first spectrum pulse timing is optimized for the production of small anions. With the exception of a few contaminant lines mainly pure aluminium cluster anions are detected. The lines are narrow indicating a low beam (translational) temperature $T_{\text {trans }}$. When optimized to larger clusters the mass spectrum in Fig. $2 b$ is obtained. As before, a broad intensity distribution without significant intensity steps or outstanding lines are measured. This finding is quite general for systems like $\mathrm{Al}_{n}, \mathrm{Cu}_{n}$, $\mathrm{Ag}_{n}, \mathrm{Ni}_{n}, \mathrm{~Pb}_{n}[7,8]$, independent of the cluster's charge. The envelopes of such intensity distributions can be changed by adjusting experimental parameters, but fine structures are weak or do not appear at all.

Pulsed UV laser light $(h v=3.68 \mathrm{eV})$ is utilized to detach electrons from mass-identified cluster anion bunches. In Fig. 3 a, b we present the PE spectra of negatively charged $\mathrm{Al}_{n}$ in the size range of $2-$ 25 atoms. As usually, the data are plotted as a function of electron binding energy, given by the photon energy minus the kinetic energy of the detached electron. The vertical axis represents full scale photoelectron count so that the intensities are not directly related to detachment cross sections. The energy scale is calibrated by recording PE spectra of $\mathrm{Cu}^{-}$monomers at different wavelengths, using the electron affinity (EA) value given in the literature [20]. In addition low lying excited states of neutral copper $\left({ }^{2} D_{5 / 2},{ }^{2} D_{3 / 2}\right)$ are accessible by the photon energy [21] and give two other calibration points.

From the spectra in Fig. 3 photodetachment onset energies (marked by horizontal bars) can be derived. These thresholds are estimates of upper bounds of adiabatic EAs (see Fig. 4) within an uncertainty of approx. $\pm 0.15 \mathrm{eV}$ and are not thought to be origin transitions. The degrees of geometrical change of $\mathrm{Al}_{n}^{-}$ upon neutralization is not known, i.e., no information is yet available about the difference between vertical and adiabatic electron affinities. Beside the thresholds the positions of the first maxima (which roughly correspond to the energies of maximum overlap between
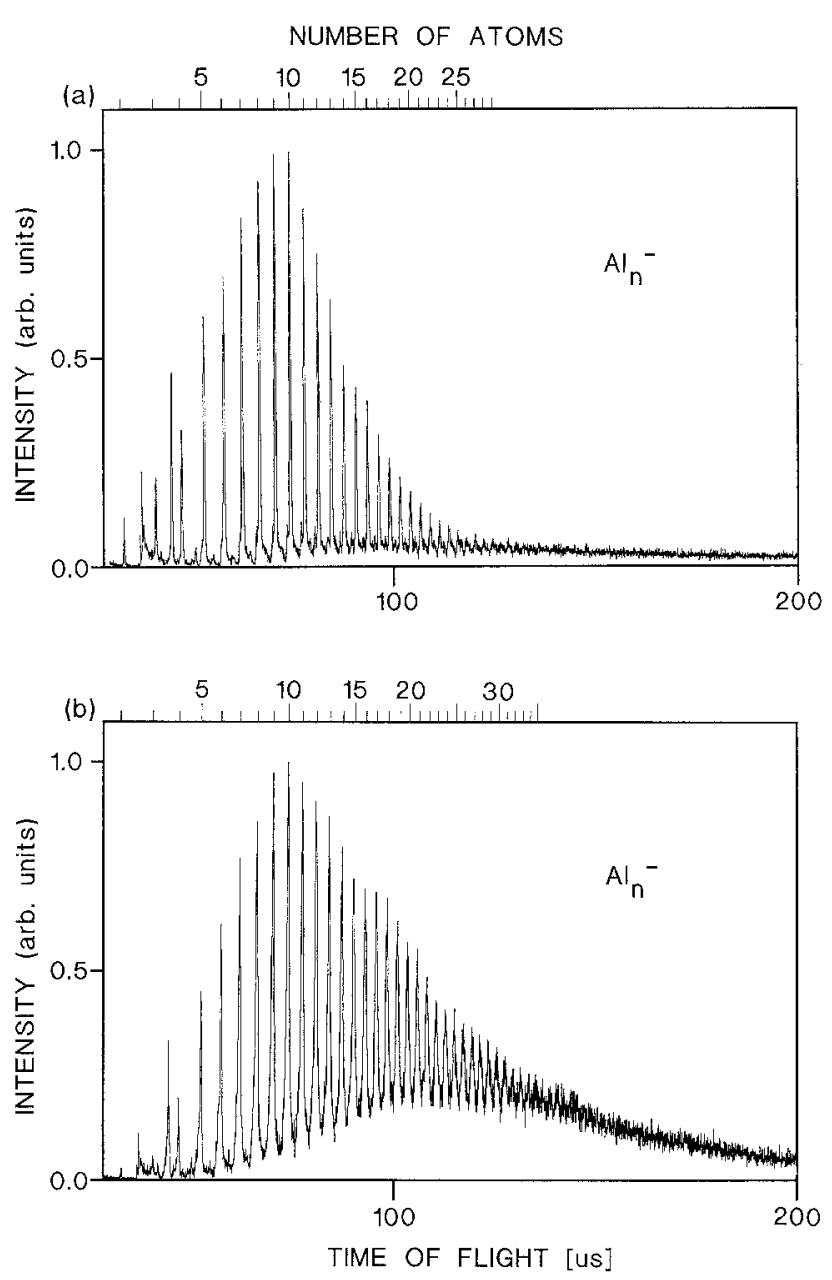

Fig. 2a and b. Time-of-flight mass spectra of aluminium cluster anions, produced by direct laser vaporization (without additional ionization). The ions are analyzed colinear to the source direction, with an energy of $300 \mathrm{eV}$. In a the source is optimized for the production of small clusters, in $\mathbf{b}$ it is adjusted for medium-sized clusters

the ionic and neutral ground state wave functions) are marked (by arrows). For low $n$ the threshold and first maximum positions (see Fig. 4) show a strong increase with $n$; these values converge slowly to an electron binding energy of about 2.5 to $3.0 \mathrm{eV}$, still being far away from the bulk work function $(\phi=4.28 \mathrm{eV}[22])$. Beside the monotonous trend some outstanding high $(n=4,6,9,19,22)$ and low $(n=14$, 23) electron affinities are observed. Furthermore, in some cases the overall PE band shape changes completely when going from $n$ to $n+1$.

For the aluminium dimer and trimer, photoelectrons are analyzed also with higher resolution at a photon energy of $h v=2.33 \mathrm{eV}$ (Fig. $5 \mathrm{a}$, b). Both spectra show weak bands (denoted by $A$ and $D$, see also Fig. 3a) at the low energy sides of the first large peaks. In a number of scans and with different source conditions we always find a situation as shown in Fig. 5. 


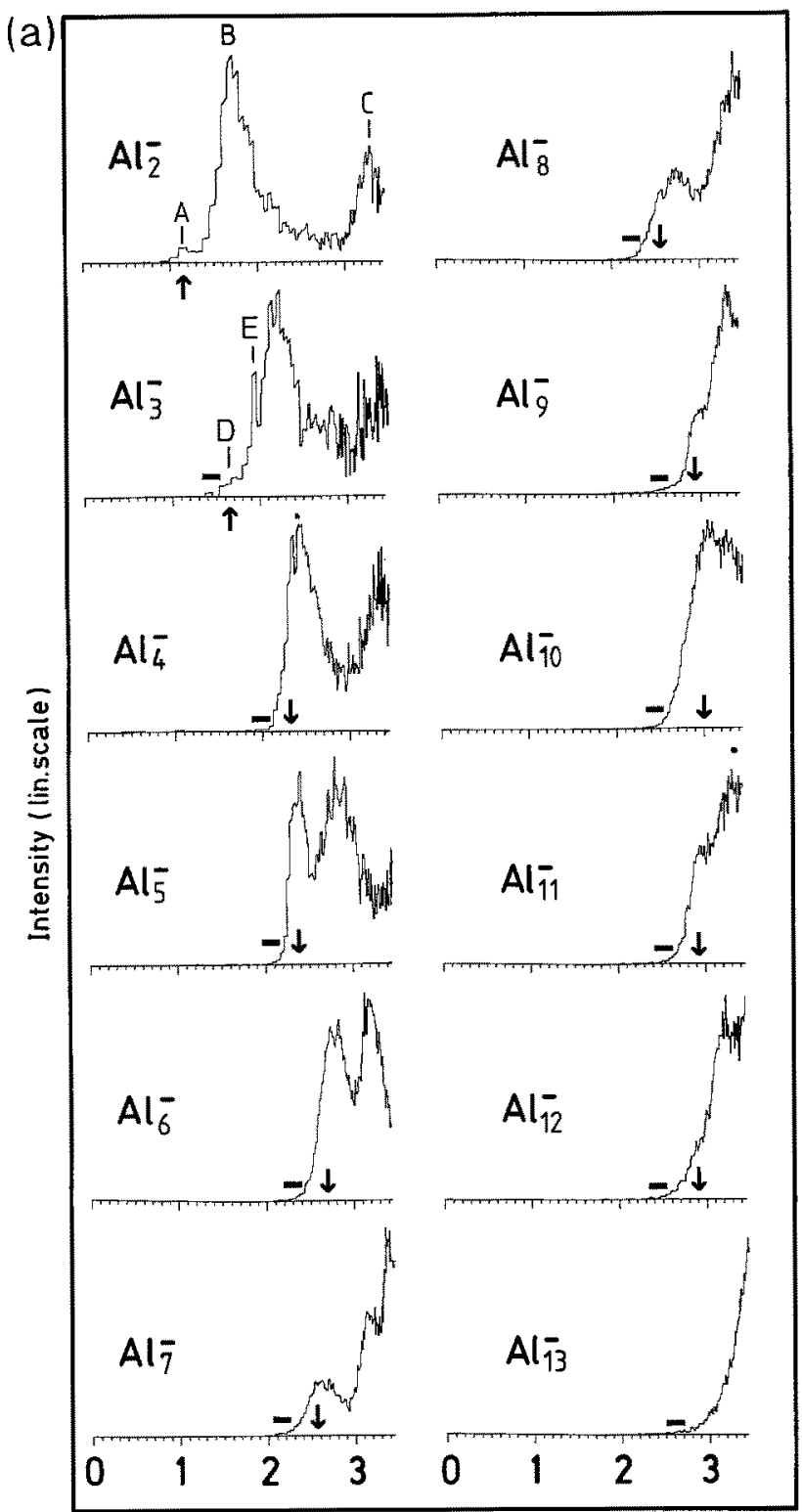

Electron binding energy [ $\mathrm{eV}]$

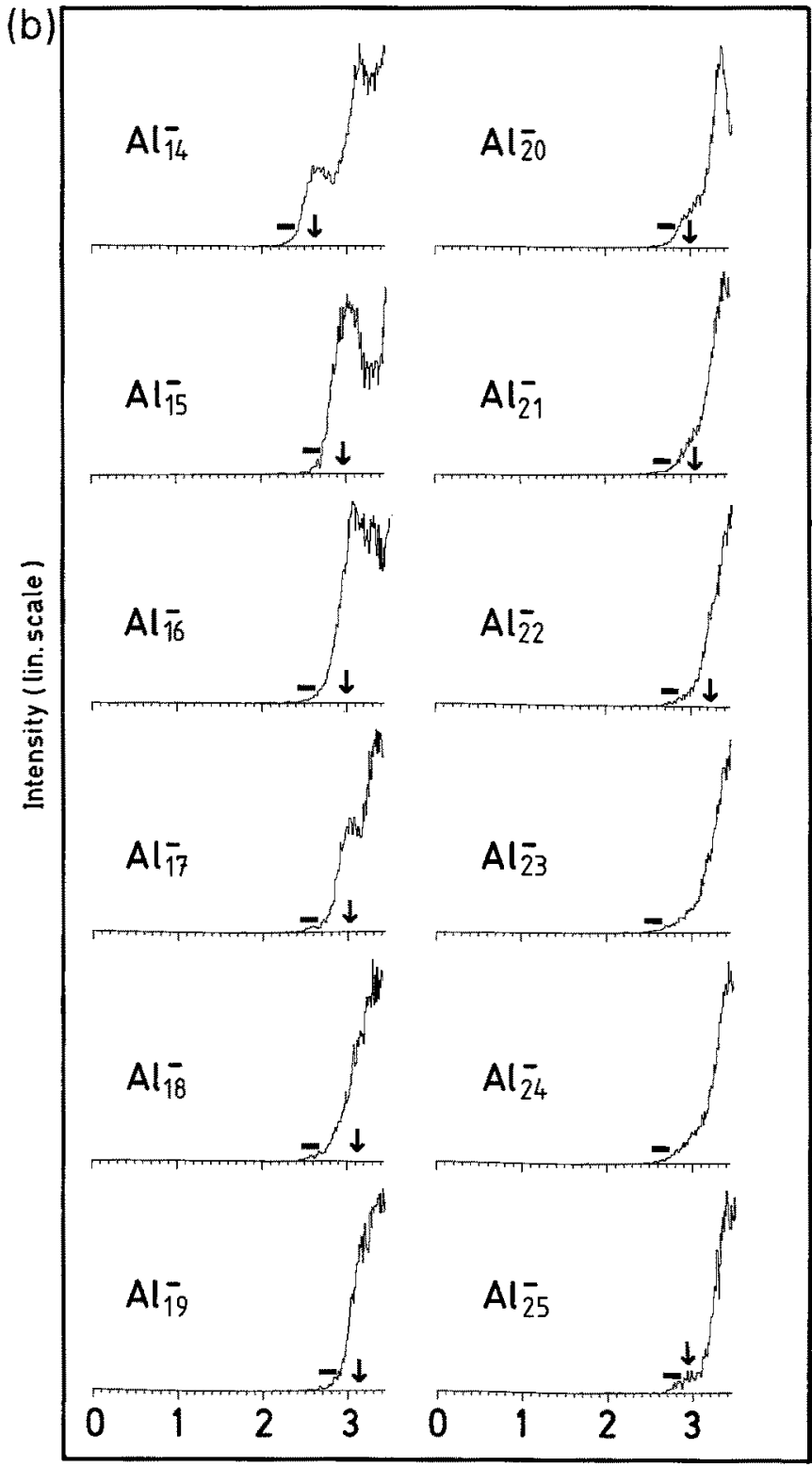

Electron binding energy $[\mathrm{eV}]$

Fig. 3a and b. Photoelectron energy spectra of $\mathrm{Al}_{n}^{-}$clusters $(n=2-25)$, at a detachment energy of $3.68 \mathrm{eV}(337 \mathrm{~nm})$. The spectra are compiled over $10^{5}$ shots, with a background signal negligible on the scale shown. Photoelectron threshold energies as estimates of upper bonds of adiabatic electron affinities are marked by horizontal bars, the arrows indicate the positions of the first maxima

The intensity ratio between peaks $A$ and $B$ (and $D$, $E$, respectively) does not change. Therefore, we interpret also these weak features as arising from ground state transitions.

\section{Discussion}

\section{A. Cluster ion production}

Laser vaporization with subsequent supersonic expansion is a powerful method for the production of cold metal cluster ions. Two features of the mass spectra in Fig. 2 are remarkable: Firstly, most of the clusters appear as pure $\mathrm{Al}_{n}^{-}$without oxides, although oxides should be stable and survive the expansion as anions (the EA of e.g. $\mathrm{Al}_{3} \mathrm{O}$ has been measured to be $1.0 \pm 0.15 \mathrm{eV}$ ). We have noted that under poor expansion conditions (low backing pressure, bad overlap between laser and valve pulse) the oxide contribution rises until the whole spectrum is governed by oxides. The cluster ion production in the extender and during the expansion seems to be determined 


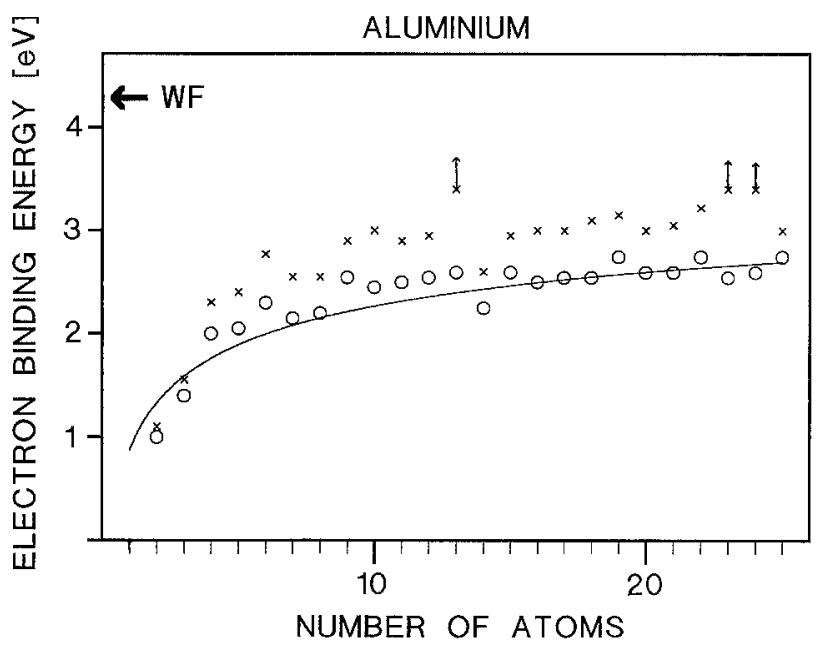

Fig. 4. Photoelectron threshold energies (circles) and the first maximum positions (crosses) as function of the number of atoms $n$. The solid line gives the electron affinities as calculated by the spherical drop model (3)

(a)

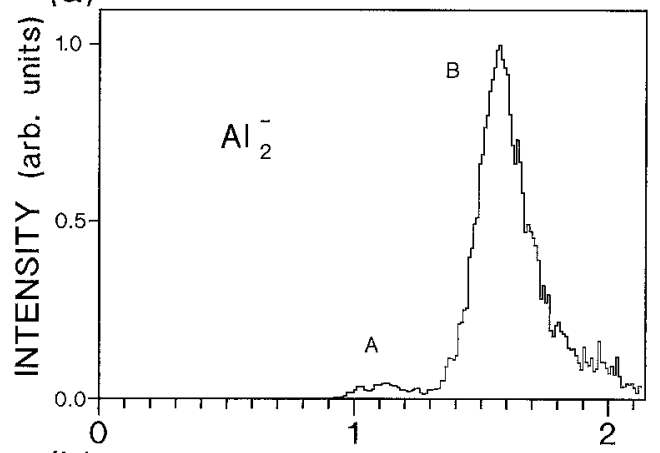

(b)

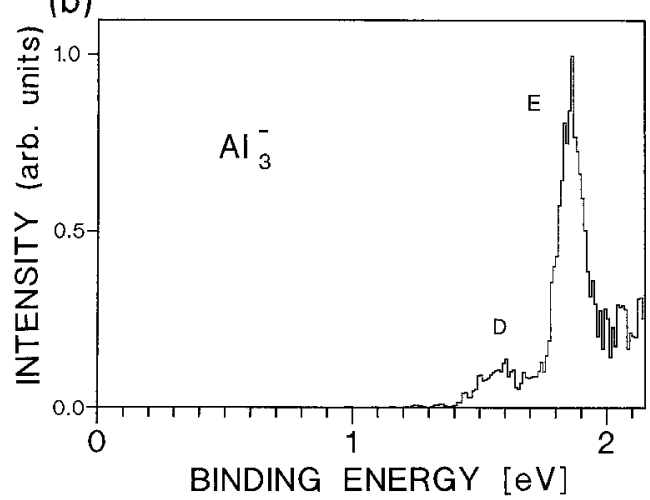

Fig. 5a and b. Photoelectron spectra of $\mathrm{Al}_{2}^{-}$a) and $\mathrm{Al}_{3}^{-}$b) at a detachment energy of $h v=2.33 \mathrm{eV}$. These spectra are taken with higher resolution and show weak maxima near the thresholds $(A$, $D$ ) which we attribute to transitions from the ionic to the neutral ground states by ion-molecule and charge exchange reactions in which the metal cluster anions survive with high abundance when the conditions have been properly chosen. We make similar observations on other metal clusters.

Secondly, the intensity distributions show one broad maximum, without pronounced discontinuities or "magic numbers", although the binding energies of these species are strongly dependent on $n$. It is well known, for example, that mass spectra of hot sputtered $\mathrm{Al}_{n}^{-}$have a huge intensity step beyond $\mathrm{Al}_{13}^{-}$ [7], for $\mathrm{Al}_{n}^{+}$such steps are found at $n=7,14,23$ [1]. Even when the clusters are produced by laser vaporization and post-ionized by light [3] steps appear at these $n$-values. In the case of sputtering we have shown [6] that such intensity anormalities are correlated with the fragmentation of hot clusters and reflect the stability of the ionic decay products. In contrast, without post-ionization metal cluster ion spectra can be produced without any intensity anormalities. In other words, the parent cluster's relative binding energy (or: "stability") is not responsible for the abundances in these spectra. Our results imply that these systems belong to the diffusion-limited regime of aggregation kinetics [23] (growth process with sticking coefficient near unity).

We note that other authors [24-26] have investigated (mainly semiconductor) cluster ions produced in a laser vaporization source, also without converting neutral clusters into ions via a secondary ionizing agent. They find strong fluctuations in the intensity distributions; these might have their origin either in a different source geometry, or in collision-induced fragmentation of the (accelerated) ions. In contrast, in our experiment the region between nozzle and skimmer is carefully shielded from electric fields in order to not disturb the supersonic expansion.

\section{B. Electronic properties of aluminium clusters}

The photoelectron spectra in Figs. 3 and 5 result from transitions of the ionic ground state to ground as well as to several excited neutral cluster states. The diffuseness of the bands can be accounted for on the basis of vibrational congestion; "hot bands" are not expected as the ions have been cooled by jet expansion. Similar broad lines have been seen by Leopold et al. for $\mathrm{Cu}_{n}^{-}[27]$ in spite of their higher instrumental resolution.

The dimer and trimer PE spectra (Fig. 5) have similar shapes, but are shifted by about $0.4 \mathrm{eV}$. Concentrating first onto the dimer, we find a weak feature (peak $A$ ) at about $1.1 \mathrm{eV}$ electron binding energy (BE), a large structure at $\mathrm{BE}=1.6 \mathrm{eV}$ (peak $B$ ), and a third 
peak around $\mathrm{BE}=3.2 \mathrm{eV}$ (peak $C$, which is only accessible at higher photon energies, see Fig. $3 a$ ). Peak $C$ could correspond to an excitation into the (spectroscopically known) $A^{3} \Sigma_{u}^{-}$-state of the neutral dimer; its term value is $17269 \mathrm{~cm}^{-1}$, or $2.1 \mathrm{eV}$ [28]. Accordingly we should find the ground-state transition $\left(\mathrm{Al}_{2}^{-} \rightarrow \mathrm{Al}_{2}{ }^{3} \Pi_{u}\right) 2.1 \mathrm{eV}$ below feature $C$, i.e. around $1 \mathrm{eV}$. Therefore, we attribute feature $A$ to the transition from ground state anion to ground state neutral dimer.

As the ions have been cooled in the supersonic jet it can be excluded that the low-energy peaks $A$ (and $D$ ) originate from excited ionic states. With the neutral dimer ground state being a triplet, the ionic ground state must either be a doublet or a quartet. Sunil et al. [29] calculated the dimer anion ground state to be a ${ }^{4} \Sigma_{g}^{-}$, Upton [9] found it to be a ${ }^{2} \Pi_{u}$. The prominent feature $B$ corresponds to one (or more) neutral excited state(s) with a term value of about $3500 \mathrm{~cm}^{-1}$. Only singlet excited states are theoretically known in this area and have been assigned to be ${ }^{1} \Sigma_{\mathrm{g}}^{+},{ }^{1} \Pi,{ }^{1} \Delta_{\mathrm{g}}[29-31]$; therefore, the only possibility for the ionic dimer ground state is to be a doublet.

With these assignments in mind we can also solve the problem of the large intensity difference between feature $A$ and $B$ : Starting from a doublet ionic ground state, one paired electron must be ionized to form the ${ }^{3} \Pi_{u}$ (or perhaps the ${ }^{3} \Sigma_{g}$ ) neutral state; such a process rises the spin multiplicity (two unpaired electrons) and is known to occur with a smaller line strength compared to one in which an unpaired electron is ionized.

The electronic ground state of the trimer anion has been calculated to be a singulet with an EA of $1.43 \mathrm{eV} \mathrm{[9]} \mathrm{(which} \mathrm{is} \mathrm{close} \mathrm{to} \mathrm{our} \mathrm{threshold} \mathrm{value}$ of $1.4 \pm 0.15 \mathrm{eV}$ ) to form either a doublet or quartet neutral cluster. When the lowest neutral state is also that with the highest spin multiplicity, feature $D$ in Fig. $5 \mathrm{~b}$ might stem from a correlated process which has a low line strength. Petterson et al. [11] suggest a ${ }^{4} A_{2}$ neutral ground state as it had also been assigned in an ESR experiment on $\mathrm{Al}_{3}$ [32]. However, magnetic deflection experiments [3] find a doublet ground state. This discrepancy could have its origin in different cluster production conditions where lowlying excited states (or isomers) play a role.

In the PE spectra of larger aluminium clusters (see Fig. 3) several bands are resolved showing one or two distinct gaps which shift to higher energies with increasing $n$. For $n>14$ (Fig. 3 b) the gaps show a tendency to close. This more or less smooth development has irregularities at $n=6,13$, and $14 . \mathrm{Al}_{13}^{-}$, e.g., has a high EA value, and the first band vanished or is shifted to energies around $3.5 \mathrm{eV}$. Therefore, the ex- cess electron occupies a strongly bound orbital, i.e. $\mathrm{Al}_{13}^{-}$should be an especially stable anion. The PE spectrum of $\mathrm{Al}_{14}^{-}$, on the other hand, has a distinct low-energy band. We interpret neutral $\mathrm{Al}_{14}$ being a stable closed shell species where the excess electron, forming the anion, occupies a new shell. In this case the band gap is a direct measure of the energy difference between the highest occupied and the lowest unoccupied molecular orbital (HOMO-LUMO gap). This interpretation is fairly conclusive as the EAtrend has a strong break at $n=14$ (see Fig. 4). For the other clusters also showing a low-energy band such an interpretation might be misleading: the mere existence of a more or less separated peak at low energies is not an unambiguous proof of a closed shell neutral cluster.

The PE thresholds as well as the positions of the first maxima show a slow convergence to the bulk work function WF as shown in Fig. 4. We apply a simple electrostatic model in which the image charge interaction between an electron and a conducting neutral sphere leads to the following prediction for the electron affinities as function of the sphere's radius $R$ [33]:

$\mathrm{EA}(R)=\mathrm{WF}-(5 / 8) *\left(e^{2} / R\right)$.

This classical spherical drop model is in rough agreement with observed $\mathrm{Cu}_{n}$ electron affinities when $R$ is given by $R=r * n^{1 / 3}+r_{0}$, with $r$ being the mean half distance between the atoms, and $r_{0}$ a correction taking into account the "spilling-out" of the electron density over the cluster radius [27]. We choose the work function $\mathrm{WF}=4.28 \mathrm{eV}$ and the mean half atomic distance $(r=1.58 \AA)$ as given by their bulk values. Assuming $r_{0}=2 a_{0}$ ( $a_{0}$ the Bohr radius) the $n$-dependence of the EA can be calculated as shown by the solid line in Fig. 4. The threshold values are found to be in good agreement with the simple model, especially for large $n$; also the energetic positions of the first maxima follow the same trend.

To obtain an estimate of monovalent metal cluster binding energies "jellium" calculations turn out to be useful [34]: In this model the atomic valence electrons are filled into a potential made up by the homogeneously distributed positive charge of the cores. Enhanced binding energies are obtained for closed-shell structures. Chou et al. [13] expected that the same model works for other simple metals like magnesium and aluminium, since bulk properties of these metals can be described adequately by the nearly free electron model, and the details of the screened ionic potential only introduce minor effects. Aluminium is trivalent and the calculated shell closings are rarely integer multiples of three; therefore, the effects might 
not be so distinct as in the case of, e.g., copper [1, $27,35]$. Within the scope of this experiment only one exact shell closing appears at $\mathrm{Al}_{13}^{-}$(40 electrons). The corresponding PE spectrum shows a slightly enhanced value for the electron threshold and a strong first maximum. The spectral difference to $\mathrm{Al}_{14}^{-}$is pronounced, a clear sign of a shell effect. Another closing is expected around $n=22$ ( 68 electrons); the threshold value for $\mathrm{Al}_{22}^{-}$is slightly enhanced although the number of electrons (67) matches not exactly with the calculated closing. Note that also positive aluminium cluster ions show distinct shell effects as has been demonstrated for ionization potentials [2] and for unimolecular decomposition $[1,6]$. We expect the jellium model to give information also on low-lying excited electronic states of neutral $\mathrm{Al}_{n}$ which are responsible for the PE band structure in Figs. 3 and 5.

Beyond the comparatively simple jellium model, elaborate quantum chemical calculations have been performed on the electronic properties of small $\mathrm{Al}_{n}$ taking into account the geometric arrangements of their ionic cores [9-12]. All authors agree in that the $\mathrm{Al}$ clusters are characterized by a number of lowlying electronic excited states as results of the different spin multiplicities and/or the $s^{2} p^{1}$ atomic valence configuration. Even for the dimer about twenty singlet and triplet states might be combined from two ground-state $\mathrm{Al}$ atoms. All of these states are expected to lie within $1 \ldots 4 \mathrm{eV}$ of the molecular ground state. This rich array of states breaks into manifolds of $\Sigma$, $\Pi$, and $\Delta$ symmetry. Going to higher $n$, the complexity will not be reduced; therefore, it is not surprising that the theoretical studies are mostly in disagreement as far as the state ordering is concerned.

In view of these problems simple models will still continue to play am important role. Of course, in the small cluster limit the jellium model is not expected to apply because of the lack of atomic structure in the potential. To improve the situation, Upton [9] introduced a perturbation of the smooth symmetric potential of the droplet model by the true electronnuclear attraction potential which removes the level degeneracies and alters the level filling order. The effect of the perturbation diminishes with increasing cluster size. This perturbed electron droplet model yields ionic as well as neutral electronic states; the resulting electron affinities $(n<6)$ are (with exception of the dimer) in agreement with our photoelectron thresholds.

A further problem is found in the question to which extent are theoretical calculations directly applicable to real experimental conditions: very likely, in an experiment clusters tend to appear in a variety of isomers or "nearly degenerate" electronic states. Therefore, one prerequisite for bringing more light into the situation is the experimental investigation of clusters at very low temperatures, thereby hoping to obtain clusters in a defined state. Experiments as reported here may show a way to solve the problem, although spectral congestion (which might partly be diminished by better instrumental resolution) will make a quantitative and detailed interpretation of valence photoelectron bands to challenge for experimentalists as well as theorists.

We thank F. Engelke, A. Ding, and Spectra-Physics for technical support. This work has been supported by the Deutsche Forschungsgemeinschaft (DFG).

\section{References}

1. Begemann, W., Dreihöfer, S., Meiwes-Broer, K.H., Lutz, H.O.: Z. Phys. D - Atoms, Molecules and Clusters 3, 183 (1986)

2. Jarrold, M.F., Bower, J.E., Krauss, J.S.: J. Chem. Phys. 86, 3876 (1987)

3. Cox, D.M., Trevor, D.J., Whetten, R.L., Rohlfing, E.A., Kaldor, A.: J. Chem. Phys. 84, 4651 (1986)

4. Dietz, T.G., Duncan, M.A., Powers, D.E., Smalley, R.E.: J. Chem. Phys. 74, 6511 (1981)

5. Ruatta, S.A., Hanley, L., Anderson, S.L.: Chem. Phys. Lett. 137, 5 (1987)

6. Begemann, W., Meiwes-Broer, K.H., Lutz, H.O.: Phys. Rev. Lett. 56, $2248(1986)$

7. Begemann, W., Dreihöfer, S., Ganteför, G., Siekmann, H.R., Meiwes-Broer, K.H., Lutz, H.O.: In: Elemental and molecular clusters. Springer Series in Materials Science, Vol. 6, p. 230. Berlin, Heidelberg, New York: Springer 1988

8. Ganteför, G., Meiwes-Broer, K.H., Lutz, H.O.: Phys. Rev. A37, $2716(1988)$

9. Upton, T.H.: Phys. Rev. Lett. 56, 2168 (1986); J. Chem. Phys. 86, 7054 (1987)

10. Pacchioni, G., Koutecky, J.: Ber. Bunsenges. Phys. Chem. 88, 242 (1984)

11. Pettersson, L.G.M., Bauschlichter, C.W. Jr., Halicioglu, T.: J. Chem. Phys. 87, 2205 (1987)

12. Hermann, K., Hass, H.J., Bagus, P.S.: Z. Phys. D - Atoms, Molecules and Clusters 3, 159 (1986)

13. Chou, M.Y., Cohen, M.L.: Phys. Lett. 113A, 420 (1986)

14. Bondybey, V.E., English, J.H.: J. Chem. Phys. 74, 6978 (1981)

15. Siekmann, H.R., Ganteför, G., Gausa, M., Meiwes-Broer, K.H., Lutz, H.O.: (to be published)

16. Posey, L.A., Deluca, M.J., Johnson, M.A.: Chem. Phys. Lett. 131, 170 (1986)

17. Kruit, P., Read, F.H.: J. Phys. E16, 313 (1983)

18. Ganteför, G., Gausa, M., Meiwes-Broer, K.H., Lutz, H.O.: Faraday Disc. 86 (in press)

19. Chesnovsky, O., Yang, S.H., Pettiette, C.L., Craycraft, M.J., Smalley, R.E.: Rev. Sci. Instrum. 58, 2131 (1987)

20. Hotop, H., Lineberger, W.C.: J. Phys. Chem. Ref. Data 4, 539 (1975)

21. Moore, C.F.: Atomic energy levels. Natl. Bur. Stand. Ref. Data Ser. 35, Washington (1971)

22. Weast, R.C. (ed.): Handbook of chemistry and physics. Boca Raton, Florida: CRC Press 1986

23. Bernholc, J., Phillips, J.C.: Phys. Rev. B33, 7395 (1986)

24. Bloomfield, L.A., Geusic, M.E., Freeman, R.R., Brown, W.L.; Chem. Phys. Lett. 121, 33 (1985) 
25. Liu, Y., Zhang, Q.-L., Tittel, F.K., Curl, R.F., Smalley, R.E.: J. Chem. Phys. 85, 7434 (1986)

26. Geusic, M.E., Freemann, R.R., Duncan, M.A.: J. Chem. Phys. 88, 163 (1988)

27. Leopold, D.G., Ho, J.H., Lineberger, W.C.: J. Chem. Phys. 86, 1715 (1987)

28. Ginter, D., Ginter, M., Innes, K.: Astrophys. J. 139, 365 (1963)

29. Sunil, K.K., Jordan, K.D.: (to be published)

30. Basch, H., Stevens, W.J., Krauss, M.: Chem. Phys. Lett. 109, $212(1984)$

31. Upton, T.H.: J. Phys. Chem. 90,754 (1986)
32. Howard, J.A., Sutcliffe, R., Tse, J.S., Dahmane, H., Mile, B.: J. Phys. Chem. 89, 3595 (1985)

33. Wood, D.M.: Phys. Rev. Lett. 46, 749 (1981)

34a. Hermann, A., Schumacher, E., Wöste, L.: J. Chem. Phys. 68, $2327(1978)$

34b. Ekardt, W.; Phys, Rev. B29, 1558 (1984)

34c. Knight, W., Clemenger, K., de Heer, W., Saunders, W., Chou, M., Cohen, M.: Phys. Rev. Lett. 52, 2141 (1984)

35. Begemann, W., Dreihöfer, S., Meiwes-Broer, K.H., Lutz, H.O.: In: The physics and chemistry of small clusters. NATO ASI Ser. B, 158, 269 (1987) 